\title{
Motivation: Origins of the Extrinsic/Intrinsic Debate
}

\author{
John H Hummel* \\ Department of Psychological Science, University of North Georgia, Dahlonega, Georgia-30597, USA
}

Received: November 01, 2013; Accepted: January 28, 2014; Published: January 30, 2014

*Corresponding author: John H Hummel, Department of Psychological Science, University of North Georgia, 82 College Circle, Dahlonega, Georgia-30597, USA, Tel: 706-864-1444; E-mail: jhhummel@ung.edu

\begin{abstract}
For over three decades cognitive researchers have studied the overjustification effect, indicating that positively reinforcing behaviors that are intrinsically motivated decreases such behaviors. As a result, some in education advocate that positive reinforcement be limited in educational settings. These studies are reviewed as well as several single-subject studies on the effects of extrinsically reinforcing intrinsically motivated behaviors. While group studies often detect an overjustification effect, it is short-lived and does not occur when positive reinforcement is implemented correctly. Positive reinforcement is a necessary component during the acquisition and maintenance phases of learning.
\end{abstract}

\section{Introduction}

In the early 1970s a series of studies were conducted by social psychologists that, collectively, form the corpus for what is known as the "overjustification effect." The overjustification effect [1] occurs when measurable environmental events (e.g., money, stars, promises, etc.) are used as contingent consequences for responses that have not been recently followed by such events. In these studies the performance of participants who experienced extrinsic reinforcement decreased as a result of their experience. The decrease in the participants' performance supposedly resulted from them evaluating why they were initially engaging in the activity. According to Deci [2], the overjustification effect may work in the following way:

It may be that money and closely related tangible rewards have some peculiar property associated with them which affects intrinsic motivation differently from nontangible rewards.

Specifically, it is suggested that money is frequently used as a means of "buying" services which would probably not otherwise be rendered. Perhaps, then, the presence of money as an external reward suggests to the subjects that they "should probably not render this activity without pay," that is, they should not be so intrinsically motivated to do the activity.

This could lead the subjects to a process of cognitive reevaluation of the activity from one which is motivated by the anticipation of money [2].

Not surprisingly, one of the outcomes associated with the overjustification studies was that in management circles, financial incentives lost some of their popularity for use as rewards in many applied settings. As a graduate student in the early 1970s I clearly remember professors in several of my classes explaining that money "is not a good reinforcer to use," and I never formally questioned this declaration. Instead, I just thought I was somewhat strange because money seemed to function very well, thank you, as a reward for my performance in various applied settings!

It was not until severalyears ago, though, that I was sufficiently motivated to begin formally examining the empirical basis for the overjustification effect and whether the declaration that money is not a good reward for most people was viable. The setting event for this was the publication of a book by Harvard educator Alfie Kohn. Kohn takes the position (though he paints with a broader brush than most of the authors of the overjustification studies) that parents, teachers, employers, etc. should not use praise and other extrinsic rewards because they serve as punishment for the people who receive them. In effect, extrinsic reinforcement harms the intrinsic motivation of people.

\section{They ought to want to do it}

Educators and psychologists have been debating the merits of intrinsic versus extrinsic reinforcement for over forty years [3]. Extrinsically motivated performance includes behaviors that are "...maintained by obvious environmental consequences...and behavior that occurs in the seeming absence of consequences..." [4] is classified as intrinsically motivated.

Many people (including teachers) believe that intrinsic motivation is an inner desire or motive to perform or engage in an activity because the activity, itself, is reinforcing. If one ascribes to this type of definition for intrinsic motivation, then one cannot, of course, hope to teach students to be intrinsically motivated because it is something that one either has or does not have. This is especially unfortunate (and unnecessary) because it makes the innocent child culpable for not having (or not having sufficient) intrinsic motivation to engage in academic activities on their own! However, if one revises the definition of intrinsic reinforcement in a way that permits the empirical study of student performance that is conceptually related to it, we avoid the potential error of victim bashing because we can structure 
the educational setting to support relevant student activities that are indicative of what could be labeled internal motivation.

Using an operational definition for intrinsic motivation (e.g., "activity that the student engages in without continuous or frequent extrinsic reinforcement") enables the construct to become an outcome that all educators can legitimately strive to achieve because it implicitly recognizes that (a) only a small percent of activities that are academically relevant become (after one masters them), themselves, activity reinforcers, and (b) if we do not reinforce these behaviors intermittently, the response class they represent will weaken and eventually extinguish. In fact, a powerful case can be made that not reinforcing students' (and workers, etc.) appropriate academic tasks (scholastic improvement, doing assignments, submitting work on time, etc.) at sufficiently high rates (i.e., on a rather rich intermittent schedule rather than on too lean of one) adversely affects the frequency of the appropriate behaviors and may result in concomitant decreases in other, related behaviors while serving as an establishing operation that evokes classes of misbehavior because of the matching law and negative reinforcement.

Additionally, educators often, after teaching students complex operations, end up weakening/extinguishing these skills because they switch from continuous-and/or-rich intermittent to more lean intermittent schedules without systematically considering the ratio between the amount of effort the student's response requires, and the power of the reinforcing consequence it, sometimes, triggers. If students work on their reports for numerous hours over a couple of weeks and their behavioral product (the report, etc.) results in a grade whose weight is the same as other less demanding academic work (e.g., a worksheet or short homework assignment), then the consequence for the report may not be sufficiently strong to maintain the students' efforts for the same quality of work in the future. One possible way to reduce this potential dilemma is to differentially reinforce higher rates of responding which is a likely explanation for what cognitive psychology refers to as the "seeking success" student with high achievement motivation.

Primates (including humans) engage in behaviors that can be described as exploratory and manipulative. While initially these types of behaviors were viewed as a vehicle for satisfying the organism's need to learn more about the environment, today such behaviors have been cited as evidence supporting a form of motivation called curiosity motivation (e.g., Eggen \& Kauchak [5]). Unfortunately, when the term motivation (or need) is used to explain why one engages in an activity, reification (using the term as an explanatory construct) has occurred. For example, after observing a child in a particular setting, a behaviorally oriented researcher might legitimately ask, "What motivates Jane to assemble jigsaw puzzles during playtime?" Used in this manner, the behavior analyst would first examine a multitude of environmental factors, both singly and in combination, that would empirically and parsimoniously answer the posed question. Non-behavioral researchers who also observed Jane are likely to ignore relevant external factors associated with her engaging in the puzzle assembly behaviors. Instead, they may conclude that Jane assembles puzzles because she is motivated to do so, a conclusion that does not really explain her behavior at all. (Motivation as an explanatory construct can result in more dire effects when it is used to "explain" why a behavior that should have occurred does not. Instead of establishing contingencies to increase the likelihood that an assignment will be completed, we intone that, "Jane didn't do the work because she is not motivated.")

Adequately answering the question of why a person engages in a particular activity when there are no apparent external consequences is an important issue from both theoretical and applied perspectives. If it can be objectively shown that humans frequently engage in truly intrinsically motivated behavior that are important to one's success in academic, social, and job-related pursuits, this would require significant adjustments in how we teach, raise our children, manage employees, etc., especially if the use of extrinsic contingencies undermine such performance. So far, this has not been demonstrated empirically.

At one level it is likely that all educators (and practically everyone else) would agree that it is a good thing when students engage in educationally and socially relevant endeavors because the activities are instrumental or reinforcing. Ideally we all desire that our charges do math (or social studies or science, or assembly of widgets) without obvious external consequences. As a group, educators ideally help mold students into independent lifelong learners who continue to study and learn even when such performance is not directly tied to environmental contingencies.

It is important to note that behaviors which occur because of programed external contingencies, what Dreikurs and Grey (1970) call arbitrary or logical consequences, have not, in applied settings such as classrooms, been shown to produce less valuable, powerful, or lower quality performances (Deitz, 1989) than those that are maintained by what both Dreikurs and Grey (1970) and [4] term natural reinforcement-contingencies that "are the natural and automatic results of responding" [4]. Obviously, extrinsic reinforcement is critical to successful teaching and learning, and performance in general for all people. Reinforcement drives the teaching of new information and skills, maintains existing skills and knowledge, can be used to decrease inappropriate behavior, and has even been shown to positively affect students' performance on standardized tests. Because teachers and others want to help students (and employees, etc.) to perform, "in the seeming absence of consequences"[4], the issues associated with the controversy between intrinsic and extrinsic motivation, and the research upon which these issues are based, require explication.

Are behaviors described as examples of intrinsic motivation (because they are emitted without apparent external reinforcement) really intrinsically motivated? Before invoking a construct such as intrinsic motivation, theoreticians should first exhaust more parsimonious explanations of the phenomenon. There are several less complex explanations that are, individually or in combination, available. First, the external consequences of the behavior are occurring but they are not recognized by 
observers because externally reinforcing stimuli can be subtle. As Fuqua (1984) has observed, "In many applied setting it may be difficult to identify the reinforcers maintaining a given behavior because a seemingly small proportion of human behavior comes into direct contact with easily identified, primary reinforces". Second, the external consequences may be occurring on a lean intermittent schedule and the observer may not observe the occurrences over a long enough period or settings and misses observing that some occurrences of the response do lead to contingent external consequences. Compounding this possibility is the concern that, "The more complex the behavior, the more difficult it becomes to determine the source of control and to completely eliminate the possibility of external control" [4]. Last, it is possible that the response itself has become a reinforcing activity to the person who is emitting it because the response (and skill at it) has been learned and refined as a result of external reinforcement processes (e.g., shaping and differential reinforcement). In this circumstance, the intrinsically motivated response (so characterized because it no longer requires external contingencies for maintenance) is a product of extrinsic motivation.

From a theoretical perspective the controversy over which is better, intrinsic or extrinsic reinforcement is important and interesting. However, from an applied and pragmatic point the issue is moot because most behaviors will extinguish if they do not produce an external reinforcing outcome at least every now and again, and I suspect one (among the many) reason that so many students (and workers) today do not work to their potential is a result of not being reinforced for improvement and/ or satisfactory completion of assigned tasks. Unfortunately, the results of group experimental studies conducted in laboratorylike settings e.g., $[4,6,7]$ have been used, incorrectly according to Lepper [8], as the basis for repudiating the use of reinforcement in applied setting including classrooms.

\section{Origins of the controversy}

The initial distinctions between intrinsic and extrinsic motivations were likely a result of theoretical developments in both social and humanistic psychologies of the 1950s and 1960s [3]. By the 1970s the stage was set for the original empirical studies on the relationship(s) that existed between the use of external rewards and their effects on intrinsic motivation. According to Morgan [3], "Demonstrations of the decremental effects of rewards began with the work of Deci $[2,9,6]$. According to reviews by both Morgan [3] and Dickinson [4] the numerous empirical studies that have found detrimental effects produced by the use of rewards generally shared some features. Among the commonly shared features of studies that investigated the overjustification hypothesis are the following.

First, the methodology employed reflected group research practices involving a limited number of trials where either data were collected or variables manipulated. Studies using multiple trial within-subject designs where data were collected repeatedly during baseline, treatment, and return-to-baseline phases generally did not show significant post treatment decrements.
Next, the group studies usually reflected assumptions that could have skewed the results. In such studies, activities were provided (e.g., puzzles, drawing supplies, etc.) that the researchers assumed had high intrinsic motivation for the subjects. Some subjects were told they would receive a reward at the end of the session if they engaged in the offered activity. At a second session data were collected on the activities the subjects selected. Non-behavioral investigators typically made one of two assumptions about the consequence selected as a reward for participating in the offered activity. The first was that the reward was a positive reinforcer. As Chance (1992) admonishes, to assume that a reward is a positive reinforcer is risky. A reward should be viewed as a non-validated positive reinforcer until its reinforcing value is established through a contingency analysis or some other appropriate tactic. Behavioral investigations on the external reinforcement/intrinsic motivation relationship used contingent positive reinforcers. In other cases researchers chose consequences to use as rewards that were not likely to be positive reinforcers! As Dickinson [4] cogently observed, it is illogical to conclude that reinforcement was responsible for decreases in subjects' performance when a reinforcement procedure was not a part of the intervention.

How the rewards provided in the studies were used also, obviously, affected whether there was a post-reward decrease in the subjects engaging in the target activity. Dickinson [4] has identified 4 types of contingencies used in overjustification studies: task, participation, performance, and success. In taskcontingent studies participants are told they will receive a reward for engaging in an activity. The participation-contingent studies deliver rewards when participants simply took part in the research. Both the task and participation-contingencies provided the reward without regard to how well (i.e., the quantity or quality) the participants' performed. In both the performance and success-contingencies delivery of the reward was based the performance meeting a preset criterion (performance) or exhibiting high quality or quantity (success).

Post-reward decrements have been reported in the majority of studies that have examined task-contingent rewards [6,10-16]. In contrast, decrements have not been reported in the majority of studies that have examined participation-contingent rewards (e.g., [9,17-18] or performance-contingent rewards [1,4,19-22].

Studies on success-contingent rewards consistently and uniformly report level or increased performance, a finding that is congruent with virtually all orientations within education. As Morgan [3] concludes, "The central finding emerging from the present review is that rewards can have either undermining or enhancing effects depending on circumstances". To avoid the possibility of transient, non-significant decrements in performance that may result if one chooses inappropriate consequence events or uses the events with inappropriate contingencies, one simply needs to use positive reinforcement correctly. "In other words, given current behavioral practices, the controversy surrounding the detrimental effects of extrinsic rewards is 'much ado about nothing" [4]. 
Respected educators who are not behaviorally oriented agree that in most circumstances the use of external reinforcement is a critical and necessary component of the teaching/learning process.

If a teachers smiles at, praises, or gives a certificate to a student, that recognition can be perceived as a messages (sic) from the teacher to the child: "I really appreciate the work you are doing." And the child may conclude "I get to do what I really enjoy doing, and the teacher supports me too."

If I show a child I am pleased he is eating an ice cream, it will not make him less motivated to eat ice cream in the future. The recognition is not perceived as the only reason to engage in the behavior. Thus, we should design learning tasks as intrinsically motivating as possible -- tasks the students would love to do with or without rewards. Adding recognition or rewards on top of good learning tasks will not necessarily erode intrinsic motivation. And for some students, the rewards will actually increase intrinsic motivation.

There is a body of research which shows that rewards can actually increase intrinsic motivation. In situations in which the rewards motivate students to engage in behaviors they otherwise would not, when later tested some show increases in intrinsic motivation -- they have found rewards in the behavior on their own. In this case the rewards have provided the incentive to get involved in the task, and once involved the students discover rewards intrinsic in the task.

For behavioral and non-behavioral educators, the ramifications of the intrinsic/extrinsic debate are clear and can be reduced to the following points. First, and most importantly, only a small portion of human activity-irrespective of whether the activity is innate or learned--is intrinsically reinforcing. While some of what we have learned, once mastered as a result of extrinsic contingencies, becomes a conditioned reinforcing activity, even these activities are likely to be extrinsically reinforced once in a while. In fact, as stated earlier, it is not idle speculation to conclude that a significant reason that American students do not spend as much time engaged on academically relevant tasks as do students in other countries is because the intermittent schedule maintaining the performance is too lean, the reinforcer used as a consequence is not sufficiently powerful, or both. External positive reinforcement is probably the most powerful tool available to teachers because the fabric of instruction and learning is woven almost entirely from it!

Too many teachers have been persuaded (i.e., conditioned) that (a) activities students are already doing do not have to be reinforced, and (b) reinforcing student performance which appears to be intrinsically motivated will weaken the likelihood of the performance occurring without the reinforcing consequence (the overjustification effect). The former position is faulty because it almost invariably will lead to extinction (the cessation of responding that results from no longer reinforcing a previously reinforced behavior), and the latter is not supported by research studies investigating the overjustification effect when reinforcement contingencies are used appropriately.
Based on all of the above, one can only conclude that not only should teachers and other managers use contingent positive consequences to maintain and improve/increase students' and workers performance, they must do so in order to prevent extinction. Additionally, the positive contingent consequences employed should reflect a rich intermittent schedule rather than a lean one especially when the reinforcers used are of questionable potency. For example, is the phrase, "Good job," sufficiently powerful for an assignment that took the student an hour and a half to do? And if only every sixth assignment triggered the phrase would the schedule be rich enough to maintain or strengthen the students' homework completion behaviors? It seems that the use of praise (an easy to use though relatively weak type of social reinforcer), when used with in conjunction with success-contingent [3] social and academic tasks, should be used by teachers with almost one-to-one correspondence between the target performance and the praise.

\section{References}

1. Reiss S, Sushinsky LW (1975) Overjustification, competing responses, and the acquisition of intrinsic interest. Journal of Personality and Social Psychology 31: 1116-1125.

2. Deci EL (1971) Effects of externally mediated rewards on intrinsic motivation. Journal of Personality and Social Psychology 18: 105-115.

3. Morgan M (1984) Reward-induced decrements and increments in intrinsic motivation. Review of Educational Research 54: 5-30.

4. Dickinson AM (1989) The detrimental effect of extrinsic reinforcement on "intrinsic motivation." Behav Anal 12: 1-15.

5. Eggen P, Kauchak D (1994) Educational psychology: classroom connections. Merrill, New York.

6. Lepper MR, Greene D,Nisbett RE (1973) Undermining children's intrinsic interest with extrinsic rewards: A test of the overjustification hypothesis. Journal of Personality and Social Psychology 28: 129-137.

7. Morgan M (1983) Decrements in intrinsic interest among rewarded and observer subjects. Child Development 54: 636-644.

8. Lepper MR (1981) Intrinsic and extrinsic motivation in children: Detrimental effects of superfluous social controls. In W.E. Collins (Ed.), Aspects of the development of competence. Lawrence Erlbaum, Hillsdale, New Jersey, pp. 155-214.

9. Deci EL (1972) Intrinsic motivation, extrinsic reinforcement, and inequity. Journal of Personality and Social Psychology 22: 113-120.

10. Anderson R, Manoogian ST, Reznick JS (1976) The undermining and enhancing of intrinsic motivation in preschool children. Journal of Personality and Social Psychology 34: 915-922.

11. Calder BJ, Staw BM (1975) Self-perception of intrinsic and extrinsic motivation. Journal of Personality and Social Psychology 31: 599-605.

12. Danner FW, Lonky E (1981) A cognitive-developmental approach to the effects of rewards on intrinsic motivation. Child Development 52: 1043-1052.

13. Green D, Lepper MR (1974) Effects of extrinsic rewards on children's subsequent intrinsic interest. Child Development 45: 1141-1145.

14. Lepper MR, Greene D (1975) Turning play into work: Effects of adult surveillance and extrinsic rewards on children's intrinsic motivation. Journal of Personality and Social Psychology 31: 479-486. 
15. Mc Graw, Kenneth O, Fiala J (1982) Undermining the Zeigarnik effect: Another hidden cost of reward. Journal of Personality 50: 58-66.

16. Ross M, Karniol R, Rothstein M (1976) Reward contingency and intrinsic motivation in children: A test of the delay of gratification hypothesis. Journal of Personality and Social Psychology 33: 442-447.

17. Pinder CC (1976) Additivity versus nonadditivity of intrinsic and extrinsic incentives: Implications for work motivation, performance, and attitudes. Journal of Applied Psychology 61: 693-700.

18. Swann WB, Pittman TS (1977) Initiating play activity of children: The moderating influence of verbal cues on intrinsic motivation. Child Development 48: 1128-1132.
19. Farr JL (1976) Task characteristics, reward contingency, and intrinsic motivation. Organizational Behavior and Human Performance 16: 294-307.

20. Farr JL , Vance R J, McIntyre RM (1977) Further examinations of the relationship between reward contingency and intrinsic motivation. Organizational Behavior and Human Performance 20: 31-53.

21. Feingold BD, Mahoney MJ (1975) Reinforcement effects on intrinsic interest: Undermining the overjustification hypothesis. Behavior Therapy 6: 367-377.

22.Vasta R, Stirpe LA (1979) Reinforcement effects on three measures of children's interest in math. Behavior Modification 3: 223-244. 